\title{
The Evolution of Cuspy Triaxial Galaxies Harboring Central Black Holes
}

\section{Citation}

Holley\#Bockelmann, Kelly, J. Christopher Mihos, Steinn Sigurdsson, Lars Hernquist, and Colin Norman. 2002. "The Evolution of Cuspy Triaxial Galaxies Harboring Central Black Holes." The Astrophysical Journal 567 (2): 817-27. https://doi.org/10.1086/338683.

\section{Permanent link}

http://nrs.harvard.edu/urn-3:HUL.InstRepos:41381854

\section{Terms of Use}

This article was downloaded from Harvard University's DASH repository, and is made available under the terms and conditions applicable to Other Posted Material, as set forth at http:// nrs.harvard.edu/urn-3:HUL.InstRepos:dash.current.terms-of-use\#LAA

\section{Share Your Story}

The Harvard community has made this article openly available.

Please share how this access benefits you. Submit a story.

Accessibility 


\title{
THE EVOLUTION OF CUSPY TRIAXIAL GALAXIES HARBORING CENTRAL BLACK HOLES
}

\author{
Kelly Holley-Bockelmann, ${ }^{1}$ J. Christopher Mihos, ${ }^{2}$ Steinn Sigurdsson, ${ }^{3}$, Lars \\ HERNQUIST $^{4}$, AND COLIN NORMAN ${ }^{5}$ \\ Draft version December 12, 2018
}

\begin{abstract}
We use numerical simulations to study the evolution of triaxial elliptical galaxies with central black holes. In contrast to earlier studies which used galaxy models with central density "cores," our galaxies have steep central cusps, as observed in real ellipticals. As a black hole grows in these cuspy triaxial galaxies, the inner regions become rounder owing to chaos induced in the orbital families which populate the model. At larger radii, however, the models maintain their triaxiality, and orbital analyses show that centrophilic orbits there resist stochasticity over many dynamical times. While black hole induced evolution is strong in the inner regions of these galaxies, and reaches out beyond the nominal "sphere of influence" of a black hole, our simulations do not show evidence for a rapid global transformation of the host. The triaxiality of observed elliptical galaxies is therefore not inconsistent with the presence of supermassive black holes at their centers.
\end{abstract}

Subject headings: galaxies: elliptical, galaxies: kinematics and dynamics, galaxies: structure, methods: N-body simulations

\section{INTRODUCTION}

Observations indicate that most, and perhaps all elliptical galaxies harbor supermassive black holes at their centers (e.g. Gebhardt et al. 2000; Richstone et al. 1998; but see Gebhardt et al. 2001b). In fact, best-fit models of black hole demography indicate that roughly $97 \%$ of ellipticals contain such black holes (Magorrian et al. 1998). The masses of these black holes seem to be correlated with properties of the host bulge; current dynamical estimates have yielded black hole masses of order $0.005 M_{\text {bulge }}$ (Kormendy \& Richstone 1995; Magorrian et al. 1998; van der Marel 1999). There is also apparently a trend between black hole mass and galaxy velocity dispersion, implying that there is a Fundamental Plane even in the four-dimensional space defined by $\left[\log M_{\mathrm{BH}}, \log L, \log \sigma_{e}, \log R_{e}\right.$ ] (Gebhardt et al. 2001a; Ferrarese \& Merritt 2001). These correlations suggest that galaxy formation and the formation of central black holes are deeply connected.

It is believed that a central supermassive black hole can profoundly influence the evolution of its host galaxy. Massive black holes dominate the galactic potential inside a radius of influence $r_{\mathrm{BH}} \approx 100\left(M_{\mathrm{BH}, 9} / \sigma_{0,200}^{2}\right)$ pc where $M_{\mathrm{BH}, 9}$ is the black hole mass in units of $10^{9} M_{\odot}$ and $\sigma_{0,200}$ is the central velocity dispersion in units of $200 \mathrm{~km} / \mathrm{sec}$. Within $r<r_{\mathrm{BH}}$, the three-dimensional structure and phase space of a galaxy will be determined largely by the black hole. Moreover, the effects of a central black hole can reach far beyond $r_{\mathrm{BH}}$. Owing to discrete encounters with individual stars, central black holes will "wander," in a manner akin to molecular Brownian motion (see, e.g. Chatterjee, Hernquist \& Loeb [2001] for a recent analysis of this phenomenon), effectively increasing $r_{\mathrm{BH}}$. During galaxy can- nibalism, supermassive black holes disrupt even dense lowmass companions in radial encounters (Holley-Bockelmann \& Richstone 2000; Merritt \& Cruz 2001) and may tidally torque debris into a nuclear disk following mergers from eccentric orbits (Holley-Bockelmann \& Richstone 2000). Black hole binaries created during galaxy mergers may explain the flat density profiles seen in the inner regions of the largest ellipticals (Makino \& Ebisuzaki 1996; Quinlan \& Hernquist 1997).

Central black holes can induce secular evolution as well. According to one long-standing suggestion, the growth of a massive black hole in a triaxial potential can destabilize centrophilic box orbits through stochastic diffusion, driving the global shape of a galaxy towards axisymmetry in a few crossing times (Gerhard \& Binney 1985; Norman, May, \& van Albada 1985; Merritt \& Quinlan 1998; Wachlin \& Ferraz-Mello 1998; Valluri \& Merritt 1998). If this picture of black hole induced evolution away from triaxiality is correct, it will have serious ramifications for our understanding of the fueling of active galactic nuclei (AGN) and the observed correlations between the properties of black holes and their host galaxies. Indeed, such evolution could lead to a self-regulation of AGN activity and black hole growth in ellipticals. If triaxiality is linked to AGN fueling, as a consequence of gas being unable to settle into closed orbits in such a potential (Norman \& Silk 1983), then an evolution towards axisymmetry would naturally lead to a reduction in the feeding of a central black hole. Such a scenario might also explain, at least in part, the correlation between black hole and bulge mass in early type galaxies (Valluri \& Merritt 1998).

While theoretical arguments paint a compelling picture for a link between central black holes and the loss of tri-

\footnotetext{
${ }^{1}$ Department of Astronomy, University of Massachusetts, Amherst, MA, 01002, kelly@shrike.astro.umass.edu

${ }^{2}$ Department of Astronomy, Case Western Reserve University, 10900 Euclid Ave, Cleveland, OH 44106, hos@burro.astr.cwru.edu

${ }^{3}$ Department of Astronomy and Astrophysics, Penn State University, 525 Davey Lab, University Park, PA 16802, steinn@astro.psu.edu

${ }^{4}$ Department of Astronomy, Harvard University, 60 Garden St, Cambridge, MA 02138, lhernqui@cfa.harvard.edu

${ }^{5}$ Department of Physics and Astronomy, Johns Hopkins University, Baltimore, MD 02138, norman@stsci.edu
} 
axiality in galaxies, observational support remains somewhat problematic, owing to the difficulty of inferring the three dimensional structure of galaxies from their projected properties. However, recent studies indicate that at least some luminous ellipticals are triaxial (Binney 1976; Franx et al. 1991; Tremblay \& Merritt 1995; Bak \& Statler 2000). The triaxiality of these systems indicates either that they contain at most low mass black holes (insufficient to drive evolution) or that the coupling between black holes and galaxy shape is not well understood. The former possibility appears to be inconsistent with the notion that luminous AGN are preferentially found in luminous ellipticals.

These considerations emphasize the need for realistic models of galaxies containing central black holes. Earlier theoretical studies have employed either triaxial models with constant density cores (Norman et al. 1985; Merritt \& Quinlan 1998) or spherical models with central density cusps (e.g. Sigurdsson et al. 1995). Neither of these two choices is entirely satisfactory. Observations of ellipticals made with the Hubble Space Telescope show that these galaxies typically have central density cusps $\propto r^{-\gamma}$ with $\gamma=0.5-2$ (Lauer et al. 1995). Galaxies with density cusps support different stellar orbits than e.g. $\gamma=0$ core models (Gerheard \& Binney 1985; Gerhard 1986; Pfenniger \& de Zeeuw 1989; Schwarzschild 1993; de Zeeuw 1996; Merritt 1999; Holley-Bockelmann et al. 2001), altering the response of a galaxy to the growth of a black hole. Likewise, spherical galaxies will support different orbital families than triaxial ones, whether or not they have central density cusps. While simulations employing spherical models have demonstrated the growth of black hole induced cusps and the polarization of the velocity ellipsoid in such objects (Sigurdsson et al. 1995), they of course have little to say about the evolution of triaxial ellipticals.

To address these shortcomings, we present a study of black hole growth in triaxial elliptical galaxies with central density cusps, using the "adiabatic squeezing" technique described in Holley-Bockelmann et al. (2001; hereafter, "Paper 1") to generate models with prescribed shapes and cusps that are stable for many dynamical times. Using Nbody simulations, we then adiabatically grow a black hole in these galaxies over several crossing times. We characterize the orbital families which populate the models, and find that many of the highly bound boxes, boxlets, and eccentric tubes are transformed into chaotic orbits - a clear signature of the influence of the spherical central potential in a larger, nearly unchanged, triaxial figure.

The paper is organized as follows. Section 2 summarizes our technique for generating a stable triaxial model of a galaxy, with subsequent growth of a central black hole. Section 3 shows the outcome of black hole growth in a model with central density cusp $\gamma=1.0$ and initial half mass axis ratios $b / a, c / a=0.85,0.7$, and discusses the structural changes occurring in this model as a result of the growth of a black hole of mass $M_{\mathrm{BH}}=0.01 M_{\mathrm{gal}}$. Section 4 explores the evolution of the orbital population induced by the black hole, and Section 5 provides conclusions.

\section{MODELING TECHNIQUE}

The "adiabatic squeezing" technique for generating triaxial models is discussed in detail in Paper 1. To summarize, we begin with, e.g. a spherical Hernquist (1990) model, having a density profile

$$
\rho(r)=\frac{M}{2 \pi} \frac{a}{r} \frac{1}{(r+a)^{3}},
$$

where $M$ is the total mass and $a$ is a scale length. We generate an N-body realization of this spherical model using the multimass scheme of Sigurdsson et al. (1995), so that particles have a mass which is roughly inversely proportional to their pericentric radius. This technique gives a finer sampling of the phase space of tightly bound orbits than would be feasible if equal mass particles were used throughout. We then evolve this model while adiabatically applying a drag force first along the $z$ axis (using the SCF method described below to maintain axisymmetry in the $x y$ plane) and then along the $y$ axis to yield a triaxial figure. The system is rescaled after each step so that the scale radius of the long axis, $a$, is unity. The lengths of the other axes are correlated with the strength of the drag force applied in each direction. To ensure an equilibrium final state, the model is evolved for several half-mass dynamical times without any drag forces present.

A black hole of final mass $M_{\mathrm{BH}}$ is then grown adiabatically in one of these equilibrium models over a time $t_{\mathrm{BH}}$ according to:

$$
M(t)= \begin{cases}M_{\mathrm{BH}}\left[3\left(\frac{t}{t_{\mathrm{BH}}}\right)^{2}-2\left(\frac{t}{t_{\mathrm{BH}}}\right)^{3}\right], & \text { for } t \leq t_{\mathrm{BH}} \\ M_{\mathrm{BH}}, & \text { for } t>t_{\mathrm{BH}}\end{cases}
$$

This expression ensures smooth evolution of the black hole mass, i.e. that $\dot{M}(t)=0$ at $t=0$ and at $t=t_{\mathrm{BH}}$. The potential of the black hole is that of a softened point mass:

$$
\Phi_{\mathrm{BH}}(r, t)=\frac{M(t)}{\sqrt{r^{2}+\epsilon_{\mathrm{BH}}^{2}}},
$$

where the softening parameter is set to $\epsilon_{\mathrm{BH}}=0.001$ in the simulations described here. This softening sets the resolution scale of our model and can be compared to the radius of influence of a $1 \%$ mass black hole, which is $r_{\mathrm{BH}} \sim 0.03$.

To ensure that black hole growth is adiabatic, we chose $t_{\mathrm{BH}}$ to be long compared to the orbital periods of stars in the inner regions of the galaxy (Sigurdsson et al. 1995). We varied the value chosen for $t_{\mathrm{BH}}$ and determined that for the examples presented here a black hole growth timescale $t_{\mathrm{BH}}=20$ is adequate. This can be compared with the dynamical times for the fiducial Hernquist sphere with $a=1$ which are $3.14,5.96$, and 8.33 at the scale radius $(r=1.0)$, effective radius $(r=1.815)$, and half-mass radius $(r=2.414)$, respectively. After the black hole has reached its final mass, the model is evolved for another 20 time units to allow the model to reach equilibrium.

The simulations were performed with a self-consistent field (SCF) code (e.g. Hernquist \& Ostriker 1992; Hernquist et al. 1995). In this approach, the density and potential of the galaxy are expanded in a set of basis functions, with the lowest order term chosen to represent the underlying density profile. The expansion coefficients are determined from the particle distribution, using $n$ radial terms and $(l, m)$ angular terms. The examples here adopted $n_{\max }=10$ and $m_{\max }=l_{\max }=6$. The $N$-body particles move in the combined potential field of the SCF expansion and the central black hole (Sigurdsson et al. 1997), and 
orbital accuracy is ensured by using a high-order Hermite integrator with variable time-stepping. The simulations were run on the T3E and the Blue Horizon at the San Diego Supercomputer Center.

\section{RESULTS OF DYNAMICAL MODELING}

We demonstrate our technique with an $\mathrm{N}=512,000$ particle Hernquist model with total mass $M=1$ and initial axis ratios $b / a=0.85$ and $c / a=0.7$, measured at the half mass radius. To this model, we add an adiabatically growing black hole of final mass $M_{\mathrm{BH}}=0.01$. If we insist that this model lies on both the global and core Fundamental Planes (Faber et al. 1997), then the central density slope of $\gamma=1$ (neglecting the influence of the black hole) fixes the absolute magnitude $\left(M_{\mathrm{V}} \approx-21.6\right)$, effective radius $\left(r_{\text {eff }} \approx 6 \mathrm{kpc}\right)$, and projected central velocity dispersion $\left(\sigma_{p} \approx 310 \mathrm{~km} / \mathrm{sec}\right)$ of the corresponding galaxy. Scaling our model to such a galaxy results in a unit length, unit velocity, and unit time corresponding to $3 \mathrm{kpc}, 1200 \mathrm{~km} / \mathrm{sec}$, and $3 \times 10^{6}$ years, respectively. Thus, in our model, the scaled half-mass dynamical timescale is $2.5 \times 10^{7}$ years.

As the black hole grows, both the cusp slope, $\gamma$, and projected central velocity dispersion, $\sigma_{p}$, increase. Figure 1 shows the cusp slope (measured at ellipsoidal radius $\log q=-1.3)$ and the projected central velocity dispersion of the model (measured at projected ellipsoidal radius $\log Q=-2.3$ ) as a function of time during and after black hole growth. After an initial rapid increase in $\gamma$ during the black hole growth phase (between $0<T<20$ ), the evolution in both $\gamma$ and $\sigma_{p}$ all but ceases, settling at the equilibrium values $\gamma \simeq 2.05, \sigma_{p} \simeq 0.7$. These results are characteristic of adiabatic black hole growth in cuspy galaxies and can be compared both to analytic estimates for adiabatic black hole growth in a spherical $\gamma=1.0$ model, which predict $\gamma=7 / 3, \sigma_{p}=0.75$ (Quinlan, Hernquist \& Sigurdsson 1995) and to the results from N-body simulations of adiabatic black hole growth in a Hernquist sphere, where $\gamma \approx 2.2$ and $\sigma \approx 0.65$ (Sigurdsson, Hernquist, \& Quinlan 1995). ${ }^{6}$ Note that these high central velocity dispersions are measured on length scales smaller than the typical minimum spatial resolution at the distance of nearby ellipticals; observable central velocity dispersions are averaged over larger apertures and tend to obscure velocity cusps.

The evolution in galaxy shape is shown in Figure 2 for the innermost $2 \%, 10 \%$, and $50 \%$ of the galaxy (by mass). Prior to black hole growth, the model has a more or less uniform shape throughout, with a slight tendency towards greater triaxiality in the center (see Figure 2 at $T=0$ and Paper 1). As the black hole grows, the inner regions quickly become rounder (Figure 2, panel a); in fact, by the time the black hole has reached full mass, the central $10 \%$ of the mass, corresponding to an ellipsoidal radius $q=\sqrt{x^{2}+(y / b)^{2}+(z / c)^{2}}<0.1$ is practically spherical with axis ratios $a: b: c=1.0: 0.95: 0.92$. The shape evolution in the outer regions is much less dramatic, however (Figure 2, panel c). Following the growth of the black hole, the model exhibits a marked shape gradient, becoming more strongly triaxial with increasing radius. This shape profile, essentially axisymmetric in the center and a rela- tively unaltered triaxial figure further out, remains stable as the system settles into equilibrium from $20<T<40$, even though the dynamical timescale here is much shorter than the integration time of the simulation. Despite the near sphericity at the center, this region is, perhaps surprisingly, still triaxial enough to influence the stellar orbital dynamics (Statler 1987; Hunter \& de Zeeuw 1992), at least in principle. We will return to this issue of orbits in the next section.

The final state of this model features several hallmarks of a black hole-embedded triaxial figure. Figure 3 shows the properties of this object as a function of ellipsoidal radius $q$ at $T=40\left(12.8 t_{\text {dyn }}\right.$ at $\left.q=1\right)$, well after the black hole has stopped growing. Figure 3a shows the $\gamma \sim 2$ density cusp induced by the black hole inside $\log q=-1$. At a larger radii $\log q>-1$, though, this plot demonstrates that the system retains the original Hernquist density profile. Figure $3 \mathrm{~b}$ shows explicitly the strong shape gradient in the model. Inside $r_{\mathrm{BH}}$, both the projected and intrinsic velocity dispersions exhibit a strong central cusp (panels c and d). In the outskirts, where the model maintains its triaxiality, the projected velocity distributions follow $\sigma_{x}>\sigma_{y}>\sigma_{z}$ in accord with a triaxial model where $a>b>c$. However, inside the cusp the projected velocity dispersions are commensurate. Interestingly, the anisotropy parameter, $\beta=1-\left\langle v_{t}^{2}\right\rangle /\left\langle v_{r}^{2}\right\rangle$, becomes negative near the black hole, as many radial orbits are given a large tangential component. This is consistent with models of stellar orbits around a black hole that is adiabatically grown, where $\beta=-0.3$ (Goodman \& Binney 1983; Quinlan et al. 1995). Exterior to the black hole's radius of influence, the system is radially anisotropic $(\beta>0)$, as expected for a triaxial galaxy.

Structurally, the most dramatic change induced in the model by the black hole is the strong shape gradient as one moves inwards (from $b, c=0.9,0.8$ at $\log q=0$ to $b, c=0.95,0.92$ at $\log q=-2)$. This significant central roundening may arise if box, boxlet, and eccentric tube orbits are lost to stochasticity near the black hole (e.g. Norman et al. 1985; Gerhard \& Binney 1985), yet survive at larger radii. However, even the nearly spherical central regions may host significantly triaxial dynamics, as pointed out by Statler (1987) and Hunter \& de Zeeuw (1992). Clearly, a more rigorous orbital analysis is warranted; we present this analysis in the next section.

\section{ORBITAL PROPERTIES}

In equilibrium, the structure of a galaxy and its orbital phase space are closely related via the Collisionless Boltzmann equation. Since the intrinsic shape of an elliptical is dictated by the time-averaged configuration space density of the orbits of its stars, the shape of the galaxy may change with time, or with radius, in response to a changing potential. Hence information can be gleaned about galactic structure through the analysis of orbit families and their subsequent stability in our model. Both twoand three-dimensional analyses are useful: planar orbits are subject to less numerical scattering and provide surfaces of section that are easy to analyze and can be compared to previous studies, while three-dimensional orbits

\footnotetext{
${ }^{6}$ The fact that our measured cusp slope is less than the analytic value of $\gamma=7 / 3$ is to be expected since our cusp slope is measured over a finite radial range near the center, and is not the asymptotic $r=0$ value.
} 
The Evolution of Cuspy Triaxial Galaxies Harboring Central Black Holes

directly trace the particle distribution and populate resonances that do not project cleanly onto a plane.

Using the automated orbit classifier described in Paper 1 , we analyzed both the two-dimensional phase space along the $x y$ and $x z$ planes (where, as always, we take $x, y$, and $z$ to be aligned with the major, intermediate, and minor axes respectively) and the three-dimensional phase space populated by the particles at $T=40$. We remind the reader that the initial state of this model (the pre-black hole stage) is the same model analyzed in Paper 1, so a direct determination of the effect of the black hole can be made by contrasting the figures in Paper 1 to those here. As before, we exploited the 8-fold symmetry of the potential to minimize noise in the particle distribution. Orbits were typed by the resonances in the dominant Fourier frequencies of the particle's motion in each plane (see Paper 1 for more information on these techniques). Following previous work, we identified stochasticity by searching for a significant change in the fundamental frequency over two time intervals (Paper 1; Valluri \& Merritt 1998). Each time interval was comprised of 50 orbital periods of a long axis tube at the energy of the orbit in question. ${ }^{7}$ Each orbit was integrated for a total of $\approx 200$ orbital times, or until a hard limit of $T=8000$. This hard limit was chosen to correspond to greater than a Hubble time of evolution in the model when it is scaled to real galactic units for a galaxy at $M_{\mathrm{V}}=-21.6$. At $3 R_{e}$ in such a galaxy, the total integration is $\approx$ two Hubble times, where each integration interval is over $11 \mathrm{Gyr}$ at this hard limit.

We have simplified our identification of strongly chaotic orbits somewhat from the method used in Paper 1. Before, we followed the convention of Valluri \& Merritt (1998) to define chaotic orbits. Specifically, an orbit was considered strongly chaotic if $\Delta f=\left|f_{1}-f_{2}\right| / f_{0} T>f_{\text {crit }}$, where $f_{1}$ and $f_{2}$ are the dominant frequencies at the first and second time intervals, $f_{0}$ is the frequency of a tube about the long axis, $\mathrm{T}$ is the time between the sampled intervals, and $f_{\text {crit }}$ is the critical threshold for the onset of a strongly chaotic orbit. For the black hole-less model, we set the following chaotic threshold: $f_{\text {crit }}=0.05 \sqrt{T / t_{200}}$. The $\sqrt{T}$ time dependence was designed to describe the diffusion of chaotic orbits as a random-walk through phase space (Valluri \& Merritt 1998). This threshold isolated orbits that were so chaotic that the orbital shape was strongly altered over two successive time intervals.

In the set of calculations for Paper 1, the precise choice of $f_{\text {crit }}$ was not important, since nearly every orbit was stable and therefore had a negligible $\Delta f$. However, adding a black hole amplifies the level of chaos in a model, yields a wide spectrum of $\Delta f \mathrm{~s}$, and requires a more careful study of chaotic thresholds. In particular, histograms of chaotic subsamples preliminarily selected with the previous criterion did not show a strong (i.e. $\sqrt{T}$ ) diffusion signal. Concerned that the rising threshold would underestimate the level of chaos in our models, we devised a new, simpler technique to identify chaotic orbits. In this paper, we identify chaos as $\Delta f=\left|f_{1}-f_{2}\right| / f_{1}>f_{\text {crit }}$, where $f_{\text {crit }}$ is a constant empirical threshold that corresponds to the 95th percentile of the $\Delta f$ distribution for the most bound sample (at $E=-1.0$ ) before the black hole is grown. For that sample, $\Delta f_{\text {crit }}=0.1$. Choosing the threshold in this manner provides us with a comparative measure of the black hole's effect on the phase space distribution. Additionally, the threshold was high enough to ensure that the orbits we have identified as chaotic did not arise from the much slower diffusion of orbits due to potential noise or integration error. We tested the sensitivity of our results by sliding the threshold from $0.5 f_{\text {crit }}$ to $2.0 f_{\text {crit }}$, and found that the percentage of chaotic orbits was unaffected in this range. In other words, chaotic orbits in this sample typically had $\Delta f$ far larger than the threshold set by any of these techniques.

\subsection{Planar orbit structure}

Figure 4 shows surfaces of section as a function of binding energy for orbits confined to the $x y$ and $x z$ planes. As in Paper 1, orbits were sampled to populate the surface of section evenly. In the inner regions $(-1.0<E<-0.65)$, the box and boxlet phase space is entirely replaced by chaotic orbits. In addition, there is an easily discernible population of eccentric tubes that are also driven chaotic - on the surface of section, these orbits occupy the high velocity boundary at a given initial position. While the tubes with larger pericenters were unaffected by chaos, eccentric tubes all sample more of the center of the potential, and are more likely to be driven chaotic. We note that the stable loops seen in these tightly bound slices are not a result of motion inside the black hole's Keplerian potential; even at $E=-1.0$, the mean ellipsoidal radius $\langle q\rangle=0.2$ is far larger than the range of influence of the black hole.

The outer regions of the model responded to the growth of the black hole in a strikingly different manner. The number of orbits identified as strongly chaotic fell dramatically compared to the inner regions, and these chaotic orbits were randomly situated in box and boxlet phase space. The 2:1 resonant boxlet ("banana") is prominent and stable in the $x z$ plane of this model, although it does not occur in the original model without a black hole (see MiraldaEscude \& Schwarzschild 1989 or Lees \& Schwarzschild 1992 for more information on boxlet nomenclature). In the $x y$ plane, the resonant orbit islands are very weak, particularly in the $E=-0.40$ slice where chaotic and regular resonant orbits seem to be mixed randomly throughout box phase space. The relative number of these scattered "boxlet" orbits is consistent with the orbit identification error of $1 \%$.

A common interpretation for the lack of strong chaos in the weakly bound orbits is that they have been integrated for far fewer dynamical times than the highly bound orbits. For example, orbits in the innermost slice (where $\langle q\rangle=0.2$ ) were integrated for $\approx 200 t_{\text {orb }}$, while orbits in the outermost slice (with $\langle q\rangle=3.9$ ) had typically reached only $\approx 50 t_{\text {orb }}$ before the hard limit set by the analysis routine. Thus, under this interpretation, there has simply been less time to observe substantial stochastic diffusion in these outermost orbits, and a longer integration might produce the same fraction of chaotic orbits as is seen in the more tightly bound set. To test this hypothesis, we integrated a subset of the lesser bound box and boxlet orbits $(E=-0.40 ;\langle q\rangle=1.3)$ in the $x z$ plane for $\approx 625$ orbital times (see Figure 5). After 200 orbital times, the orbits

\footnotetext{
${ }^{7}$ Hence, each integration interval corresponds to 200 dynamical times. We note that Paper 1 erroneously stated that each time interval corresponds to 12.5 dynamical times; however, this misstatement did not affect any of the conclusions in that paper.
} 
were as dynamically evolved as the most tightly bound orbits, but the fraction of box and boxlet phase space that had gone chaotic only reached $7 \%$ (versus $\approx 100 \%$ of the box and boxlet phase space at $E=-1.0)$. As the integration time increased to well over two Hubble times, the fraction of chaotic orbits also went up. However, although the percentage of chaotic orbits in this subset increased from $2 \%$ to $16 \%$ over the course of the experiment, even after a scaled time of 29 Gyr, many stable centrophilic orbits persist, including a population of non-resonant boxes. To test the extent of numerical error over long integrations, we also integrated the same subset of orbits (i.e. $x z$ plane, $E=-0.40$ ) from our initial triaxial model without the black hole and in this experiment, only $4 \%$ of the orbits were considered strongly chaotic well after two Hubble times. So, the chaos present in these weakly bound orbits is a real effect, not simply an artifact of the longer integration time. Furthermore, the long integration time ensured that the remaining stable box orbits were stable despite repeated passes through the black hole's sphere of influence.

\subsection{Three-dimensional orbit structure}

To study the orbital evolution induced by a central black hole, it is not sufficient to consider only two-dimensional surfaces of section. Orbits in triaxial galaxies are not simply confined to the major and minor planes; there are several important 3-D resonances which serve to support a triaxial figure and which do not project down onto an identifiable resonance in a principal plane. Furthermore, the fact that 2-D orbits are confined to a plane dampens the onset of chaos. For example, in a singular logarithmic triaxial potential, much of the chaos present is in the $3-\mathrm{D}$ orbits; this chaos is not reflected in the orbit families moving in the principle planes (e.g. Papaphilippou \& Laskar 1996).

With this in mind, we also investigated the threedimensional orbital content as traced by the particle distribution. As in Paper 1, we sorted the final particle distribution according to binding energy and binned the distribution into 9 slices of equal particle number. Figure 6 shows the percentage, by mass, of tube families versus chaotic orbits as a function of binding energy and radius. Notice that the predominant orbit families in this model are tubes, and the precipitous drop-off in the number of chaotic orbits with decreasing binding energy, as seen in the planar sample, is also reflected here.

Figure 7 presents the particle distribution on a frequency map of $f_{y} / f_{z}$ versus $f_{x} / f_{z}$ after 50 orbital times. Since the initial conditions were set by the actual N-body model, this frequency map is not evenly sampled. Furthermore, since only the initial frequencies are shown, this plot does not show orbital chaos. What is shown, however, are the resonant regions that are populated by the particles in this model. In the most bound slice $(E=-1.0)$, it is clear that nearly the entire population is composed of tube orbits, since practically all of the 40000 orbits in this slice lie along the $(0,1,-1)$ or $(1,-1,0)$ resonance lines, marking the inner long-axis tubes and short-axis tubes, respectively. In fact, the only significant area that does not lie along these lines also contains tube orbits; the clump near $\left(f_{x} / f_{z}, f_{y} / f_{z}\right)=(0.8,0.98)$, contains orbits which project to long axis tubes in the xy-plane plane and low-order boxlets in another. The majority of these tube orbits are well outside the Keplerian potential of the black hole; these tubes are instead dictated by the nearly spherical stellar potential which extends out past $\log q=-1$. To underscore this point, we note that most of these tubes in this energy slice clump near the $(1,1,1)$ resonance, suggesting that the potential is not just axisymmetric here, but nearly spherical (However we note that, by itself, a predominance of 1:1:1 resonant orbits does not necessarily mandate a spherical density distribution (Statler 1987; Hunter \& de Zeeuw 1992).)

As we move out in the model to the least tightly bound particles, although the strong presence of tube orbits remains, low order boxlets are discernible, as in the $(1,-2,1)$ resonance at $E=-0.65$, and the $(2,0,-3)$ resonance at $E=-0.2$ and -0.4 . These final two panels strongly resemble the frequency maps for the same energy slices in the model without a central black hole (see Paper 1). This, along with the lack of shape evolution beyond $\log q=0$, indicates that the effect of the black hole does not strongly alter the outer regions of the model over galactic evolutionary timescales.

The general result that more loosely bound box orbits persist even well after a Hubble time is not new. Both analytic estimates and early numerical simulations indicated that while an unsoftened black hole induces stochasticity in nearly all of the box and boxlet orbits, the loss of triaxiality is confined to the center. For example, Gerhard \& Binney (1985) modeled the disruption of planar box orbits as a series of discrete scattering events by a $M_{\mathrm{BH}}=0.02 M_{\text {core }}$ black hole and found that, in a Hubble time, box orbits extending far outside the core are unlikely to have experienced enough pericentric passes to have been substantially scattered. In a fully self-consistent N-body simulation of black hole growth in a post-collapse triaxial potential, Norman, May \& van Albada (1985) showed that a black hole causes box phase space to be replaced with fully stochastic orbits on a timescale proportional to an orbit's dynamical time. This was taken to be consistent with the black hole scattering picture, with the caveat that the small number of particles in the simulation could result in an additional numerical source of scattering by two-body relaxation.

However, recent work has suggested that the trend toward stochasticity is a more rapid and a more global phenomenon. Merritt \& Quinlan (1998) conducted selfconsistent N-body experiments of black hole growth in triaxial models, and determined that black hole masses greater than $0.003 M_{\text {gal }}$ drive the galaxy toward axisymmetry, and that global stochasticity - where the axisymmetry extends well outside the half-mass radius and evolves on the order of an orbital period - occurred at $M_{\mathrm{BH}} \approx$ $0.3 M_{\text {gal }}$. Our more subtle and local evolution is not necessarily at odds with these results. The study conducted by Merritt \& Quinlan involved galaxies which were more triaxial than ours, and which also had flat $(\gamma=0)$ central density profiles. The different density structure of the models results in different orbit populations in the two studies - the constant density cores of the Merritt \& Quinlan models are likely populated by a much higher fraction of box orbits than our more cuspy models (Statler 1987; Hunter \& de Zeeuw 1992; Arnold et al. 1994). Furthermore, the growth of a central black hole in a $\gamma=0$ model 
represents a more significant perturbation to the central potential than in our $\gamma=1$ models. Even with identical initial density profiles, though, a direct comparison is complex. For example, using Schwarzschild's (1979) method of reconstructing a $\gamma=0.5$ potential via a judicious selection of orbit libraries, Valluri \& Merritt (1998) found that slightly different initial minor axis flattenings give different results for the relative fraction of tightly bound and less bound chaotic orbits. Moreover, the degeneracy between a given physical shape and its possible orbital content makes any particular solution for the orbital behavior non-unique. It is apparent from our study, though, that there exists at least one set of orbit families that generate a triaxial figure and respond to an adiabatically growing central potential in such a way as to preserve its global shape over long timescales.

\subsection{Discussion}

It is clear that the onset of chaos in the most tightly bound orbit families leads to a rapid change in the inner structure of the model galaxies. What is less clear is the specific agent driving the chaos in the system - is it scattering by the central black hole itself, the effects of the steepened stellar density cusp, or something else entirely? One way to check if the stellar cusp is responsible for the chaos is to remove the black hole and categorize the orbits that result from the $\gamma \sim 2$ frozen stellar potential. Although this is not a self-consistent galaxy model, it is a useful tool to gauge the relative importance of the black hole to the steep stellar cusp (and its spherical stellar potential). We integrated a subset of particles in the $E=-0.65$ energy slice in the purely stellar potential for a total of $200 t_{\text {orb }}$, and classified the orbits in the manner described previously. In both the two- and three-dimensional orbital analysis, we find that rapid chaos is strongly suppressed when the black hole is removed. Therefore, it seems clear from this experiment that the steep stellar cusp is not the source of strong chaos in these models. This result is interesting in light of the work of Merritt \& Fridman (1996) which links the rapid onset of global chaos to the presence of steep $(\gamma \sim 2)$ density cusps in galaxies. Earlier work by Schwarzschild (1993), however, demonstrated the secular stability of $\gamma \sim 2$ density cusps in scale-free triaxial logarithmic potentials, more in line with our results. These different conclusions indicate that the connection between cusp slope, figure shape, and orbit stochasticity may well be extremely sensitive to the various orbit families initially present in the model.

While the presence of a black hole is necessary for driving chaos in our models, the mechanics of this process are less clear. Under a simple black hole scattering model, chaotic orbits should have more - or closer - pericentric passes within $r_{\mathrm{BH}}$ than stable ones, but this is not the case in our sample. In fact, the degree of stochasticity depended on neither the number of pericentric passes within $r_{\mathrm{BH}}$ nor on the minimum $r_{\text {peri }}$. So, while the black hole does induce strong chaos, it seems not to do so through a simple scattering process. Another possibility is that chaos is driven by the transition between the inner spherical Keplerian potential of the black hole and the outer triaxial potential of the galaxy. In this picture, weakly bound box orbits in the outskirts of the model may spend too little of their orbital period in the inflection region to be signifi- cantly perturbed, and are thus not driven stochastic at all. In our simulations, however, there was no strong correlation between $\Delta f$ and the time spent within the inflection region, leaving open the question of what drives chaos. In truth, however, the graininess of the potential makes it difficult to disentangle the subtleties of chaos from numerical noise in the potential, so that analytic studies are better suited for this question.

In the outer regions, orbits stay regular even after repeated passages near the potential center. It is possible that many of these orbits are actually chaotic orbits that are "sticky" (Siopis \& Kandrup 2000), with a diffusion timescale that is much longer than a few dynamical times. The course-grainedness of our potential seems to argue against this explanation; a course-grained potential effectively creates holes in the Arnold web (Arnold 1964) through which an otherwise confined orbit may escape. In addition, we observed the same effect in the planar orbit sample, and Arnold diffusion does not occur in 2-D potentials (Merritt \& Fridman 1996). It seems likely, then, that the regularity of these orbits is real.

Issues of orbital chaos and regularity aside, our galaxy model maintains its original degree of triaxiality on a global scale despite the presence of a massive central black hole. The orbit analysis described in $\S 4$ indicates that the model will remain relatively stable for as long as a Hubble time. It is therefore interesting to compare this stable galaxy model with observations of real elliptical galaxies.

Initially, our scaling parameters were chosen to place the model on both the global and the core fundamental planes. After the evolution in structural and kinematic properties driven by the black hole, does the model still obey these relations? In the case of the global fundamental plane, the answer is certainly yes: the changes in the velocity dispersion and radial density profiles occur only in the central regions ( $\log r<-1.5$, or $r \lesssim 100$ pc ), leaving the global properties of the model unchanged. For the core fundamental plane, the increase in central cusp slope ( from $\gamma=1$ to $\gamma \sim 2$ ) represents a significant change, leaving the model with a cusp slope which is perhaps a bit too steep for its scaled luminosity of $M_{B}=-21.6$ (see e.g., , Gebhardt et al. 1996). However, the $\gamma-M_{B}$ relationship is quite steep and shows significant scatter at intermediate luminosity (Gebhardt et al. 1996), so that this discrepancy may not be significant - ellipticals with $M_{B} \sim-21$ show cusp slopes of $\gamma=1-2$. Scaling our model to more luminous ellipticals begins to present problems, however, since adiabatic black hole growth models generically predict cusp slopes steeper than that observed in luminous ellipticals (e.g., Bahcall \& Wolf 1976; Young 1980; Goodman \& Binney 1984; Sigurdsson et al. 1995; Quinlan et al. 1995).

Turning to the issue of triaxiality in ellipticals, the triaxiality parameter of the model at the half-mass radius is $T=\left(1-b^{2}\right) /\left(1-c^{2}\right)=0.5$, with a flattening $c / a=0.8$. Unfortunately, a problem arises in defining the best sample of ellipticals with which to compare our model. To select for black hole embedded ellipticals, samples of radio-loud ellipticals might seem the best choice, but luminous ellipticals typically have a flatter cusp slope than that used our models. On the other hand, although a more general sample of ellipticals may possess a wider range of cusp slopes, including steeper cusps like that of our model, they 
may also harbor black holes at a reduced rate when compared to AGN-selected samples. Bearing these caveats in mind, we compare the structural properties of our model to the triaxiality inferred for different samples of elliptical galaxies. Using a combination of photometric and kinematic data, Bak \& Statler (2000) show that the Davies \& Birkinshaw (1988) sample of radio galaxies is characterized by a range of shapes biased towards prolate figures but that - as long as ellipticals are not disk-like rotators triaxialities like that of our model are common. In addition, the typical flattening of the galaxies in that sample is $c / a \sim 0.7$, similar to, although somewhat flatter than, our models. Studies of larger samples of ellipticals (not radio selected) also show characteristic flattening similar to that of our model (e.g. Ryden 1992; Tremblay \& Merritt 1995). While a more detailed analysis of triaxiality in black hole embedded ellipticals must await more complete data, at face value our model does represent well the observed structural properties of elliptical galaxies.

The connection between supermassive black holes and triaxiality has important consequences for both secular and hierarchical galaxy evolution models. For example, it has been suggested that if a central black hole drives its host galaxy toward axisymmetry globally and rapidly, one possible difference between an intrinsically bright elliptical (thought to be more triaxial) and a faint elliptical is that the stars in the faint elliptical, with their shorter crossing times, have had more interactions with the black hole and the galaxy is thus more dynamically evolved (Valluri \& Merritt 1998). The black hole/bulge mass relation can be explained in terms of galaxy evolution (Valluri \& Merritt 1998) as well. In this scenario, spiral galaxies begin as gas-rich disks with a small triaxial bulge. The triaxial potential supports fueling of the central black hole through material falling into it on box orbits (e.g. Norman \& Silk 1983) or by gas traveling on intersecting orbits which drive dissipation and inflow. The black hole grows until a critical mass of $\simeq$ few $\% M_{\text {gal }}$, which breaks triaxiality and strongly curtails the gas inflow. Subsequent diskdisk merging can create a elliptical galaxy, and black hole feeding ensues in this larger triaxial bulge until the critical black hole mass is achieved. In both types of galaxies, the process is the same: once the black hole mass fraction is large enough to disrupt box orbits, gas inflow is sharply diminished.

The black hole in our model induced axisymmetry out to several hundred parsecs, and resulted in a clearly observable change in the shape and structure of the galaxy on these scales. Since the transformation did not take place globally, it is tempting to say that the black hole mass/bulge mass relation observed in the current galaxy population is not simply an artifact of gas inflow in a more triaxial-shaped progenitor population. However, it is not immediately clear how the more localized axisymmetry we observed would affect gas inflow and subsequent black hole feeding. While it is true in our globally triaxial model that gas inflow from outside the half mass radius would never be entirely cut off, the behavior of the gas once it hits the axisymmetric region requires detailed gas dynamical simulations. Nonetheless, it is clear that a central supermassive black hole causes dramatic and long-lasting changes in the host galaxy over scales well outside the region in which it dominates the potential.

Finally, 21-cm observations of elliptical galaxies have revealed the presence of extended neutral hydrogen disks and rings in many ellipticals (e.g. Franx et al. 1994; van Gorkom \& Schiminovich 1997; Hibbard et al. 2001). Several authors have proposed that these structures are precursors to "disk rebuilding" in ellipticals (Schweizer 1998; van Gorkom 2001). If this scenario is correct, there must be an inward migration of gas in these systems. Triaxiality offers a mechanism to drive this inflow, but if black holes were to break triaxiality on large scales it is difficult to see how migration and disk building could occur. Our results alleviate these concerns; the fact that triaxiality is maintained in all but the inner few hundred parsecs of the galaxy would allow gas to move inwards on kiloparsec scales to perhaps begin the process of disk formation. However, the modest degree of triaxiality in the model suggests that the rebuilding timescale may be long. ${ }^{8}$

\section{SUMMARY}

Using numerical simulations, we have studied the growth of central supermassive black holes inside cuspy triaxial galaxies. Unlike previous self-consistent modeling of black hole growth in triaxial ellipticals, our calculations employ progenitor galaxy models which are both triaxial and have central density cusps typical of observed ellipticals (Holley-Bockelmann et al. 2000). Inside these progenitors, we adiabatically grow a central black hole of mass $M_{\mathrm{BH}}=0.01 M_{\mathrm{gal}}$. As the black hole grows, it induces a central cusp $(\gamma \sim 2)$ in the stellar density profile and a strong roundening of the central shape of the elliptical. However, while the effects of the black hole do extend beyond its nominal "sphere of influence", out at an effective radius the galaxy figure remains largely unchanged.

To explore the change in the orbital structure induced by the black hole, we use a combination of Fourier spectral classification and axis-crossing pattern recognition to classify the orbits present in the model. At the most tightly bound energies, the models are composed entirely of loops, short and long axis tubes, and chaotic orbits. These chaotic orbits comprise all of the box and boxlet phase space present in the original model, and even a population of eccentric tubes. The outer regions predominantly contain short axis tube orbits; however, in these outer regions, there still exists a modest phase space of boxes and boxlets which support the large-scale triaxiality of the system. Of these centrophilic orbit families, there are a substantial fraction that do not go strongly chaotic, even over thousands of dynamical times. While the presence of noise in the potential expansion limits our ability to detect mild chaos in the orbit populations, we believe that the remaining boxes and boxlets in the outer regions are stable enough to continue to support the global shape of the galaxy for a Hubble time.

While massive black holes act as agents of chaos in the inner regions of our models, they provoke a more modest response in the outer regions than expected on the basis

\footnotetext{
${ }^{8}$ This is particularly problematic for the very extended (i.e. many $R_{e}$ ) rings seen in some ellipticals. In these cases, not only is the dynamical timescale long, but the dynamics are more likely driven by the structure of the dark matter halo than by the luminous galaxy itself (e.g., Franx, van Gorkom, \& de Zeeuw 1994).
} 
of earlier studies (e.g. Norman et al. 1985; Merritt \& Quinlan 1998). However, those previous studies employed galaxy models which had flat central density "cores" and were also significantly more flattened than ours, a combination which yields a significantly higher fraction of boxes in the original orbital families than found in our model. These differences are likely the root cause of the more dramatic evolution seen in previous calculations. Our galaxy models indicate that even moderately triaxial ellipticals can host central massive black holes, in agreement with observational evidence which suggests both that black holes are ubiquitous (Magorrian et al. 1998) and that triaxiality may be common (Bak \& Statler 2000).

Our more realistic elliptical galaxy models make an excellent tool for the simulation of several unsolved problems in elliptical galaxy formation and evolution. such as the degree of disk rebuilding by large-scale gas inflow, the effect of satellite infall on the structure of the galaxy, the interaction between galaxies and their triaxial dark matter halos, the persistence of central cusps, and the formation of nuclear disks.

This work is supported through a grant of computing time from the National Partnership for Advanced Computational Infrastructure and the San Diego Supercomputer Center, and by NASA through grant NAG5-7019. JCM is also supported by an NSF Career Grant and Research Corporation Cottrell Scholarship. We wish to thank Tim de Zeeuw for the careful refereeing of this paper. We thank Rebecca Stanek for help with data analysis.

\section{REFERENCES}

Arnold, V. I., 1964, Russ. Math. Surveys, 18, 8

Arnold, R., de Zeeuw, T., \& Hunter, C., 1994, MNRAS, 271, 924

Bak, J., \& Statler, T., 2000, AJ, 120, 110

Bahcall, J.N., \& Wolf, R.A. 1976, ApJ, 209, 214

Binney, J. \& Tremaine, S. 1987, Galactic Dynamics, Princeton University Press

Binney, J. 1976, MNRAS, 177, 19

Binney, J. 1985, MNRAS, 212, 767

Chatterjee, P Hernquist, L. \& Loeb, A. 2001, ApJ, submitted astro$\mathrm{ph} / \phi 107287$

de Zeeuw, T. 1995, Gravitational Dynamics, eds O. Lahav, E. Terlevich, \& R.J. Terlevich, (Cambridge: Cambridge University Press), 1

Davies, R. L. \& Birkinshaw, M. 1988, ApJS, 68, 409

Faber, S.M. et al. 1997, AJ, 114, 1771

Ferrarese, L. \& Merritt, D. 2001, ApJ, 547, 140

Franx, M., van Gorkom, J. H., \& de Zeeuw, T. 1994, ApJ, 436, 642

Franx, M., Illingworth, G., \& de Zeeuw, T. 1991, ApJ, 383, 112

Gebhardt, K. et al. 2000, ApJ, 543, 5

Gebhardt, K. et al. 2001a, ApJ, 555, 75

Gebhardt, K. et al. 2001b, AJ, in press astro-ph/0107135

Gerhard, O.E., \& Binney, J. 1985, MNRAS, 216, 467

Gerhard, O.E. 1986, MNRAS, 219, 373

Goodman, J. \& Binney, J. 1983, MNRAS, 203, 265

Goodman, J., \& Binney, J. 1984, MNRAS, 207, 511

Hernquist, L. 1990, ApJ, 356, 359

Hernquist, L., \& Ostriker, J.P. 1992, ApJ, 386, 375

Hernquist, L., Sigurdsson, S. \& Bryan, G.L. 1995, ApJ, 446, 717

Hibbard, J.E., van Gorkom, J.H., Rupen, M.P., \& Schiminovich, D. 2001, Gas and Galaxy Evolution, ASP Conf Series 240, eds. J.E. Hibbard, M.P. Rupen, \& J.H. van Gorkom, in press.

Holley-Bockelmann, K., \& Richstone, D. 2000, ApJ, 531, 232

Holley-Bockelmann, K., et al. 2001, ApJ, 549, 862 [Paper 1]

Hunter, C. \& de Zeeuw, T. 1992, ApJ, 389,79

Kormendy, J. \& Richstone, D. 1995, ARA\&A, 33, 581
Lauer, T.R. et al. 1995, AJ, 110, 2622

Lees, J. \& Schwarzschild, M. 1992, ApJ, 384, 491

Makino, J., \& Ebisuzaki, T. 1996, ApJ, 465, 527

Magorrian, J. et al. 1998, AJ, 115, 2285

Merritt, D. \& Cruz, F. 2001, ApJ, 551, 41

Merritt, D. \& Fridman, T. 1996, ApJ, 460, 136

Merritt, D. \& Quinlan, G. 1998, ApJ, 498, 625

Merritt, D. 1999 PASP, 111, 247

Miralda-Escude, J. \& Schwarzschild, M. 1989 ApJ, 339, 752

Norman, C.A., May, A., \& van Albada, T.S. 1985, ApJ, 296, 20

Norman, C., \& Silk, J. 1983, ApJ, 266, 502

Papaphilippou, Y., \& Laskar, J. 1996, A\&A, 307, 427

Pfenniger, D., \& de Zeeuw, T. 1989, Dynamics of Dense Stellar Systems, Cambridge University Press, 81

Quinlan, G., \& Hernquist, L. 1997, New Astronomy, 2, 533

Quinlan, G., Hernquist, S., \& Sigurdsson, S. 1995, ApJ, 440, 554

Richstone, D. et al. 1998, Nature, 395, 14

Ryden, B.S. 1992, ApJ, 396, 445

Schweizer, F. 1998, Saas-Fee Advanced Course 26: Galaxies: Interactions and Induced Star Formation, 105

Sigurdsson, S., Hernquist, L., \& Quinlan, G.D. 1995, ApJ, 446, 75

Sigurdsson, S., He, B., Melhem, R. \& Hernquist, L. 1997, Comp. in Phys., 11.4, 378

Siopis, C. \& Kandrup, H. 2000, MNRAS, 319, 43

Schwarzschild, M. 1979, ApJ, 232, 236

Schwarzschild, M. 1993, ApJ, 409, 563

Statler, T. 1987, ApJ, 321, 113

Tremblay, B., \& Merritt, D. 1995, AJ, 110, 1039

Valluri, M. \& Merritt, D. 1998, ApJ, 506, 686

van der Marel, R. 1999, AJ, 117, 744

van Gorkom, J. \& Schiminovich, D. 1997, ASP Conf. Ser. 116: The

Nature of Elliptical Galaxies; 2nd Stromlo Symposium, 310

van Gorkom, J. 2001, in Galaxy Disks and Disk Galaxies, in press.

Wachlin, F. C., \& Ferraz-Mello, S. 1998, MNRAS, 298, 22

Young, P. 1980, ApJ, 242, 1232 

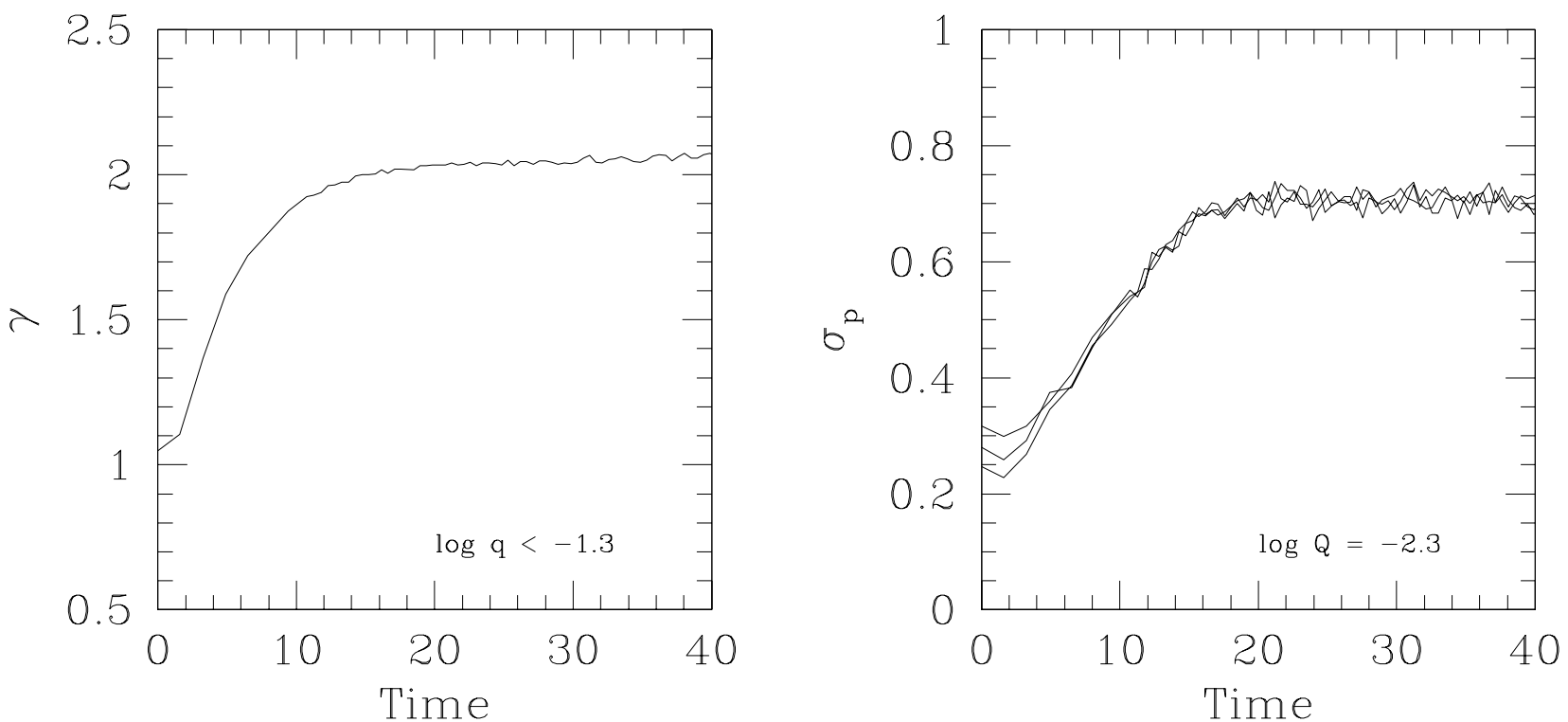

FIG. 1. - Central properties of the model galaxy during and after black hole growth. Left panel: Central density slope $\gamma$ of the model, measured at ellipsoidal radius $\log q=-1.3$, as a function of time. Right panel: Central projected velocity dispersion, measured at projected ellipsoidal radius $\log Q=-2.3$ as a function of time. 


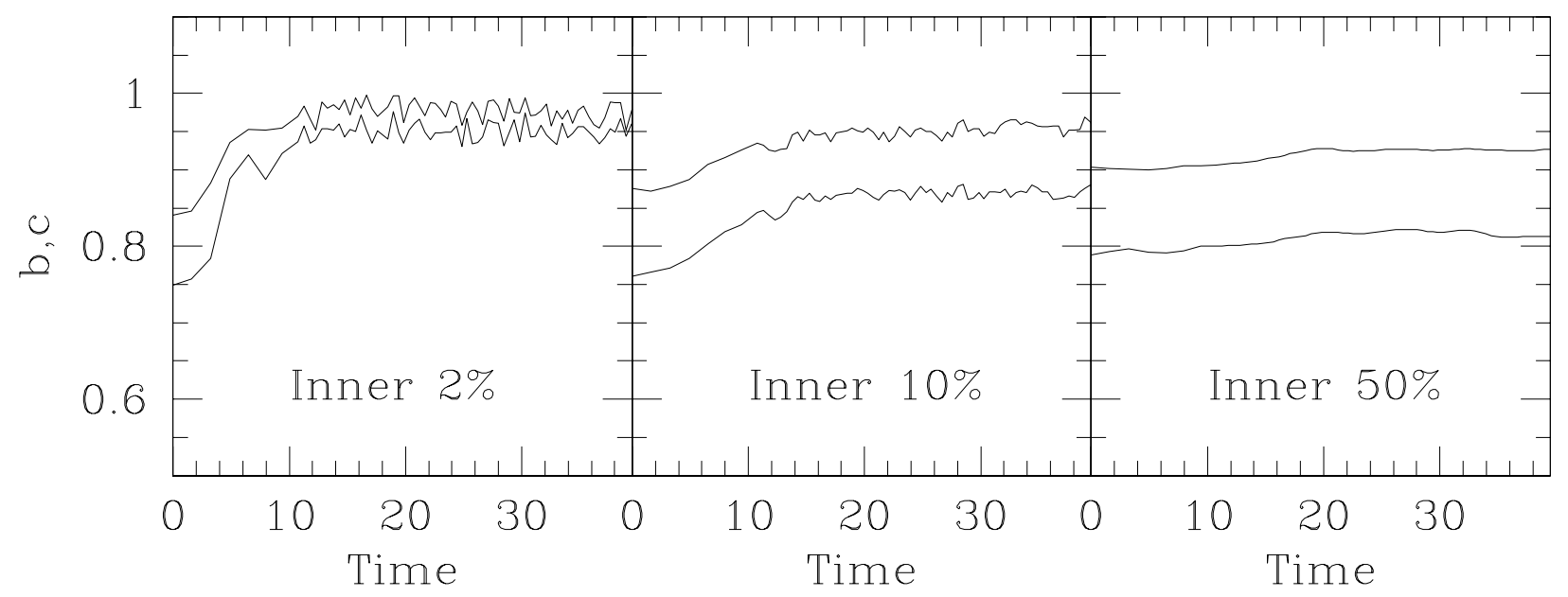

FIG. 2.- The intermediate and minor axis lengths as a function of time for particle sets binned by mass in the model galaxy. Axis lengths are calculated iteratively from the ellipsoidal density distribution using the moment of inertia tensor. (See Paper 1 for details.) 

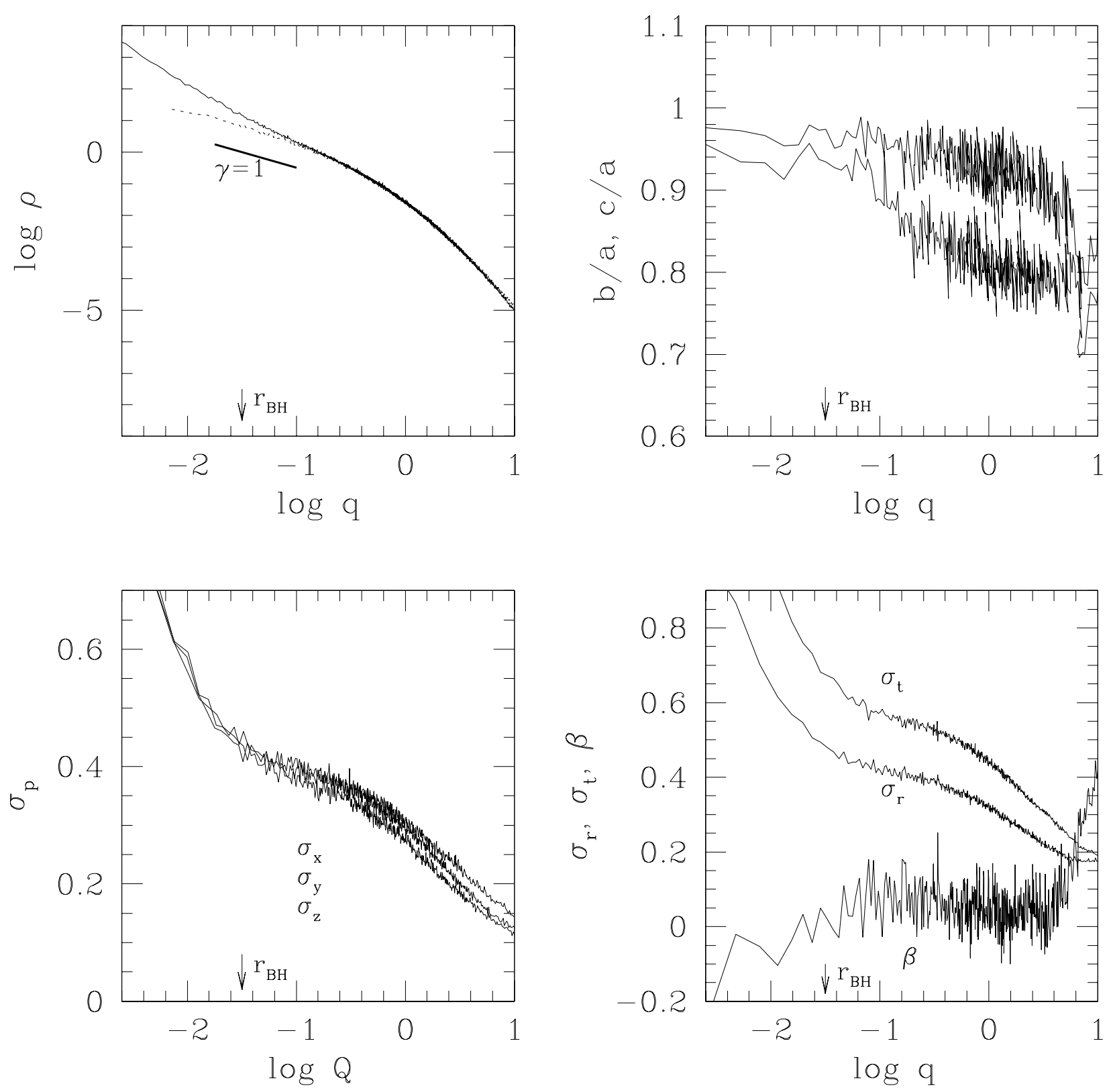

FIG. 3. - The structural and kinematic properties of the model at $T=40$. Upper left: density profile. Upper right: intermediate and minor axis lengths as a function of ellipsoidal radius. Lower left: projected velocity dispersion along the fundamental axes, as a function of projected ellipsoidal radius. Lower right: true radial and tangential velocity dispersion, and velocity anisotropy parameter, as a function of ellipsoidal radius. 


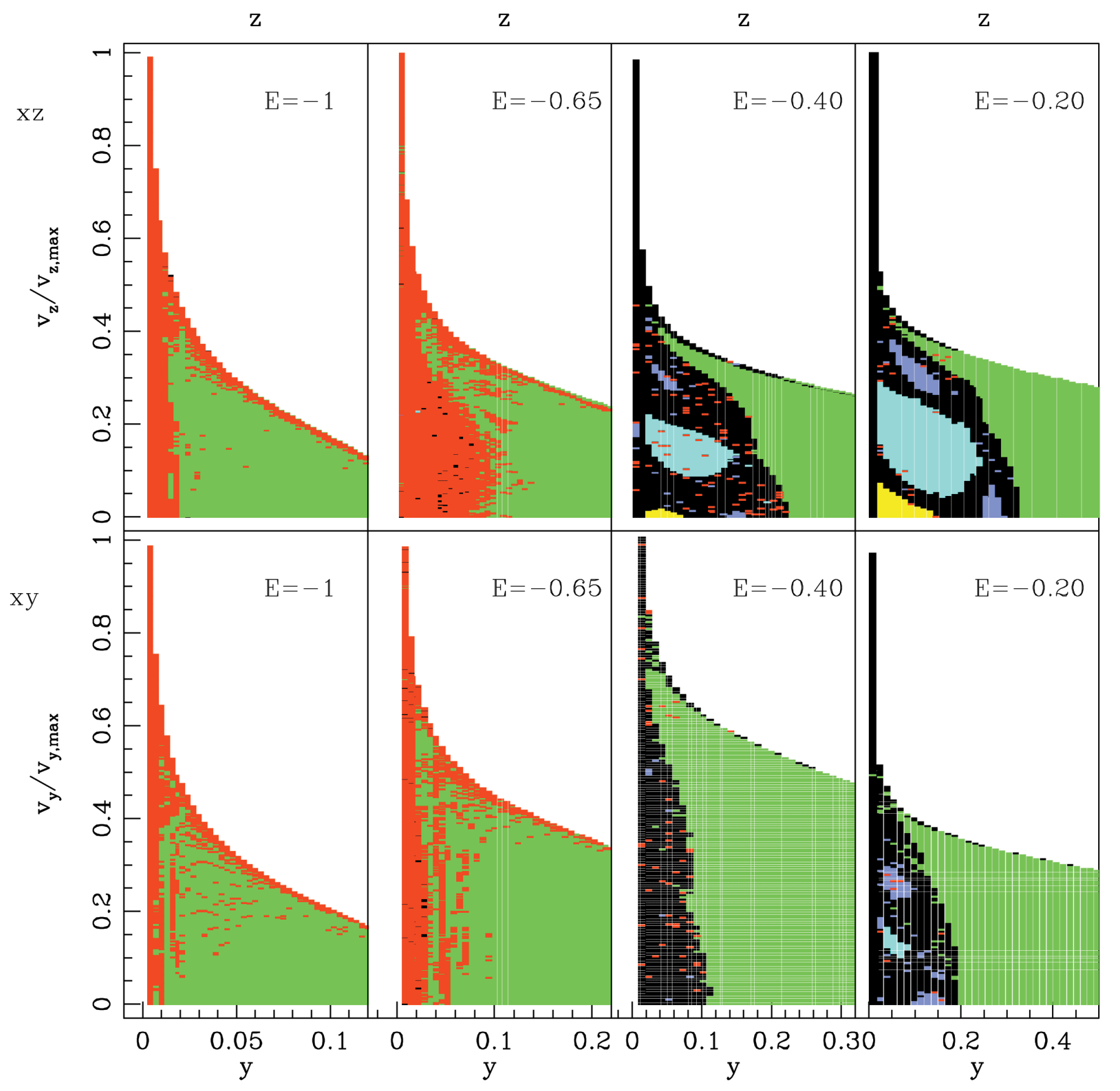

Fig. 4.- Surfaces of section for the triaxial model at $T=40$, plotted for orbital populations at different binding energies. Top: Surfaces of section for orbits in the $x z$ plane. Bottom: Surfaces of section for orbits in the $x y$ plane. Orbits are coded by color - chaotic orbits: red; loops: green; bananas: yellow; fish: blue; pretzels: aqua; higher resonance boxlets and pure boxes: black. This plot was created by taking an average of all orbit types at a particular position on the surface of section. 


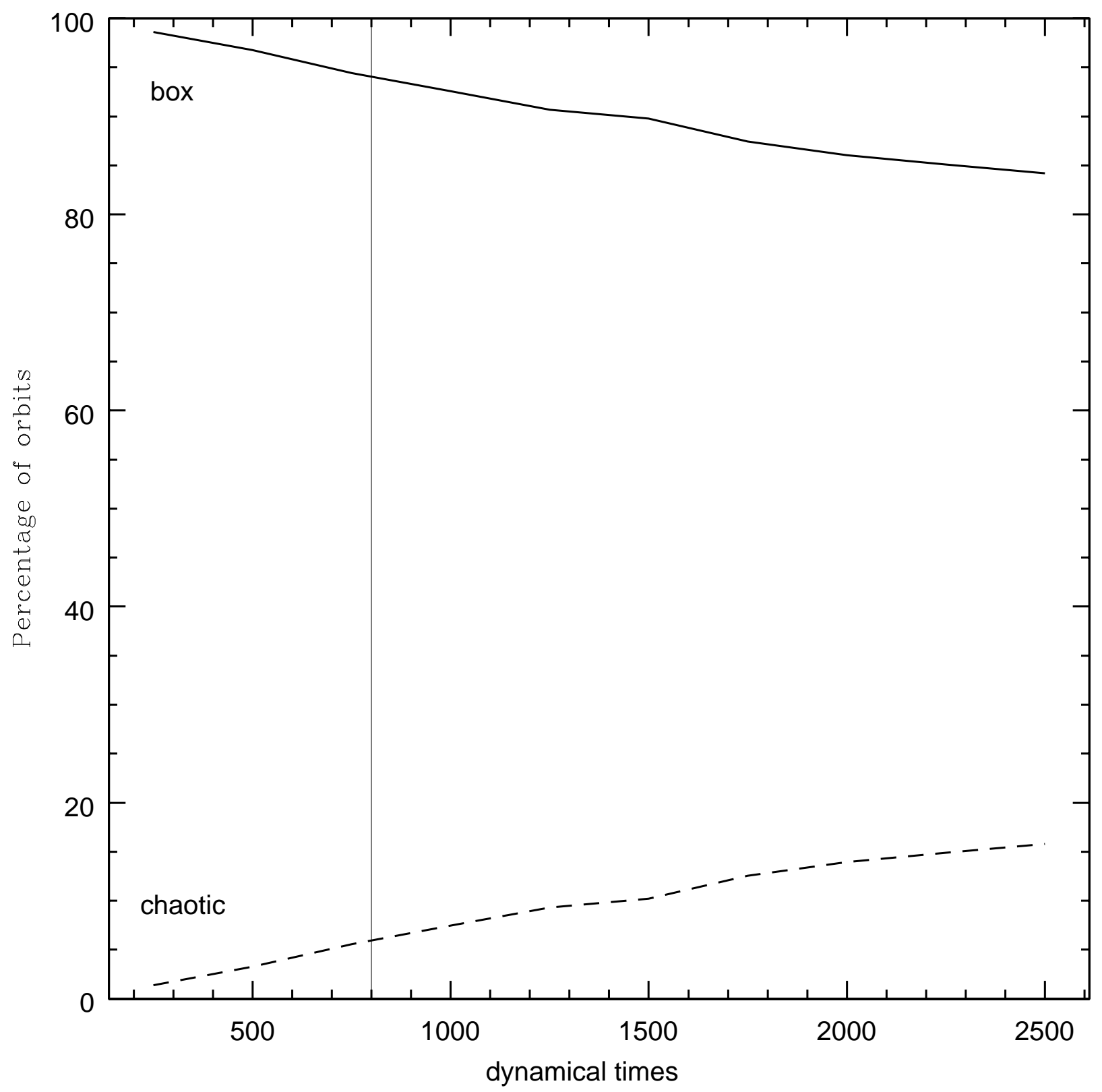

FIG. 5. - The percentage of chaotic orbits as a function of dynamical time for a subset of centrophilic planar $x z$ orbits at $E=-0.6$. 


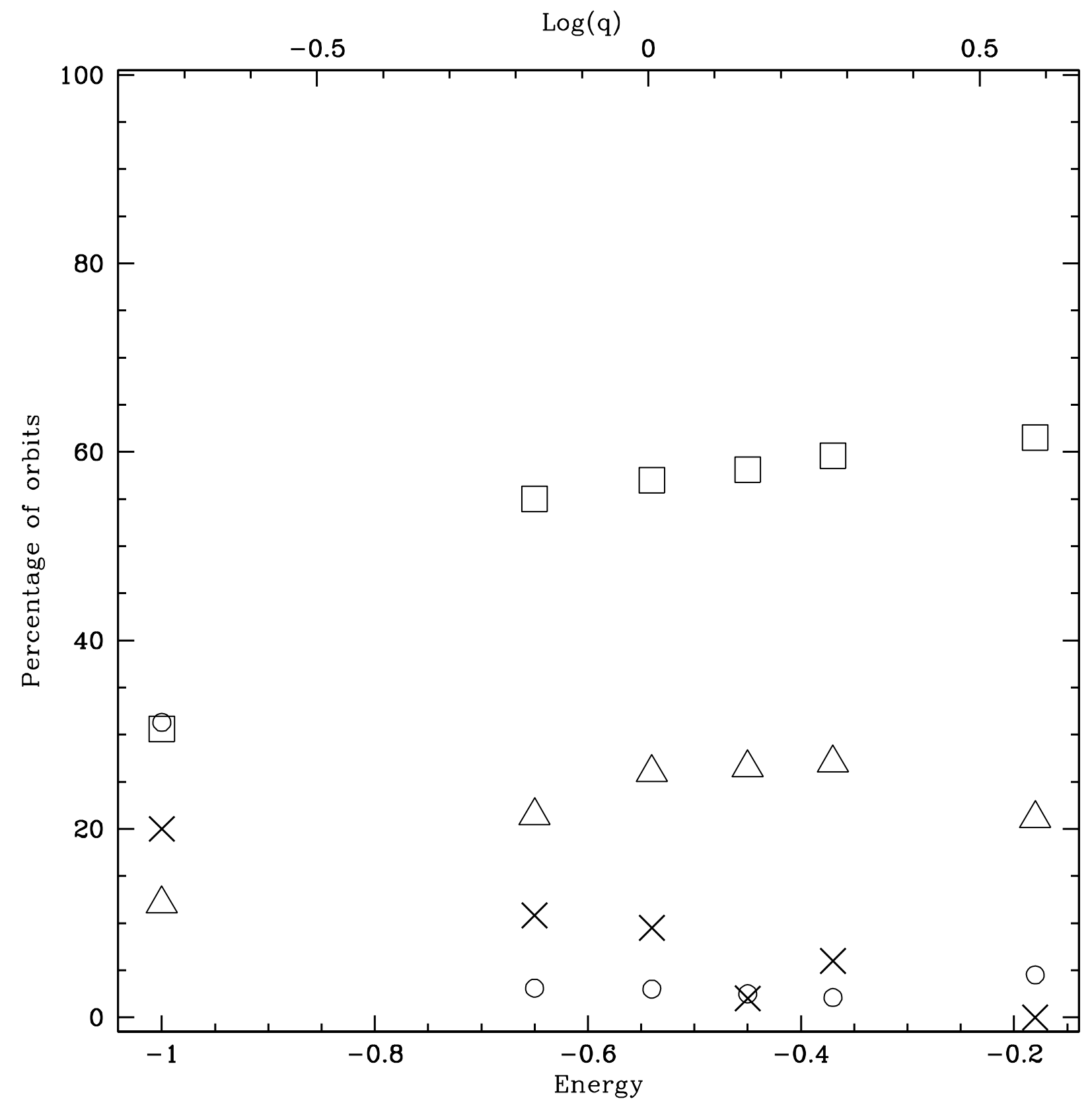

FIG. 6.- Percentage of tubes vs. chaotic orbits in the 3-D sample as a function of binding energy. Open symbols represent tubes in general; open squares represent short axis tubes, open triangles show long axis tubes, and open circles show the 1:1:1 resonance, or planar loop family. The crosses represent strongly chaotic orbits. 

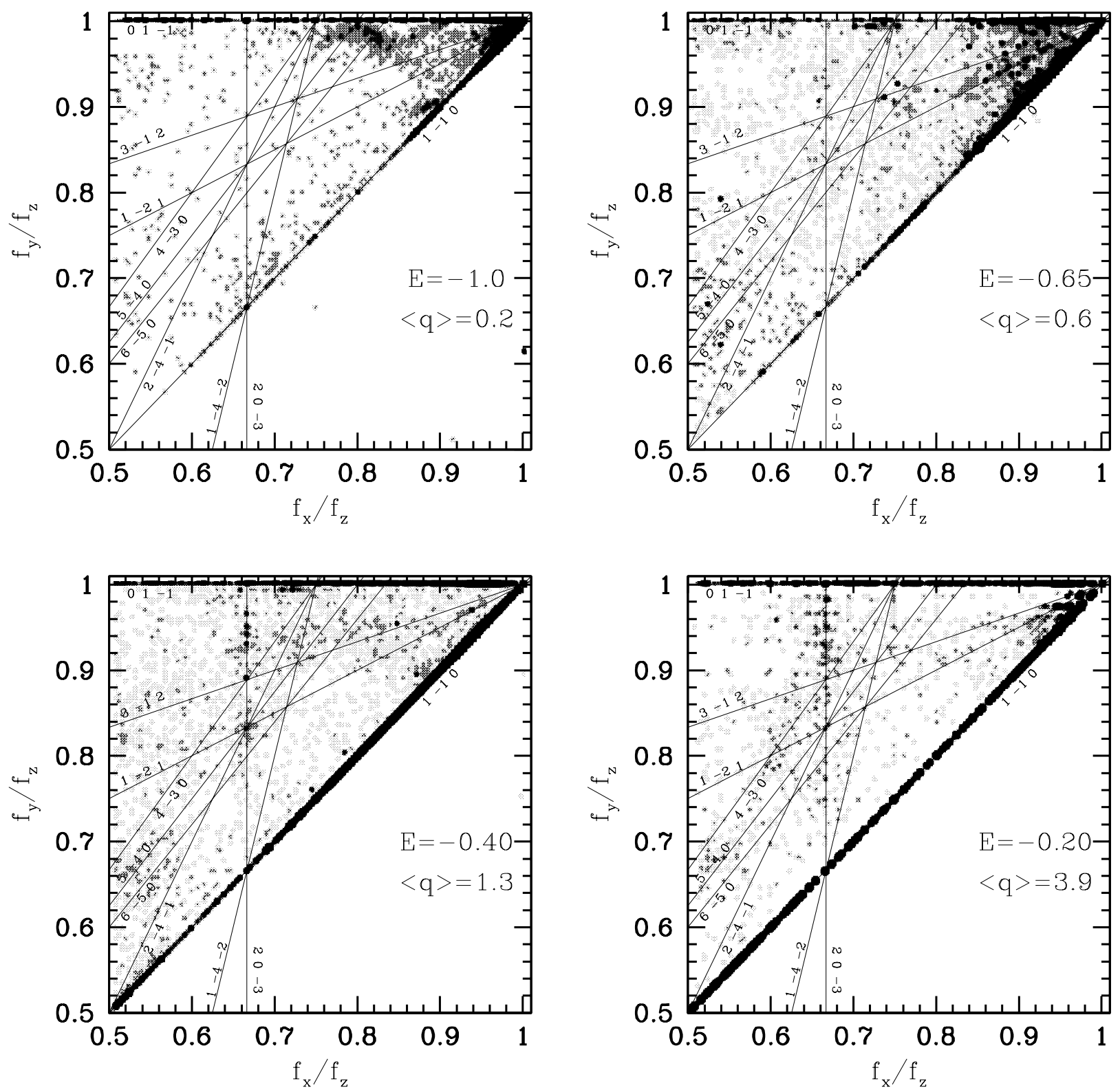

FIG. 7.- Frequency map for the triaxial model at $T=40$, plotted for orbital populations of different binding energies. The greyscale represents the number of orbits at a given frequency ratio. The lightest grey is 1 orbit, while black is greater than 50 orbits. The diagonal line corresponds to short axis tubes, and the horizontal line at $f_{y} / f_{z}=1.0$ corresponds to long axis tubes. 